\title{
A new approach for improving diagnostic accuracy in Alzheimer's disease and frontal lobe dementia utilising the intrinsic properties of the SPET dataset
}

\author{
Marco Pagani1, 2, Vassili A. Kovalev³, 4, Roger Lundqvist ${ }^{5}$, Hans Jacobsson², 6, Stig A. Larsson², Lennart Thurfjell5 \\ 1 Institute of Cognitive Sciences and Technology, CNR, Rome, Italy \\ 2 Section of Nuclear Medicine, Karolinska Hospital/Institute, Stockholm, Sweden \\ 3 Institute of Engineering Cybernetics, Belarus National Academy of Sciences, Minsk, Belarus \\ ${ }^{4}$ Max-Planck Institute of Cognitive Neuroscience, Leipzig, Germany \\ ${ }^{5}$ Applied Medical Imaging, Uppsala, Sweden \\ ${ }^{6}$ Department of Radiology, Karolinska Hospital, Stockholm, Sweden \\ Published online: 19 December 2003 \\ (C) Springer-Verlag 2003
}

Eur J Nucl Med Mol Imaging (2004) 31:307

DOI 10.1007/s00259-003-1434-4

Marco Pagani (

Institute of Cognitive Sciences and Technology, CNR, Rome, Italy e-mail: marco_00198@yahoo.com

Fax: +39-06-824737

The online version of the original article can be found at http://dx.doi.org/10.1007/s00259-003-1196-Z

\section{Eur J Nucl Med Mol Imaging (2003) $30: 1481-1488$}

The first affiliation of Marco Pagani was omitted by mistake in the printed version. Please see the complete and correct affiliations above. 